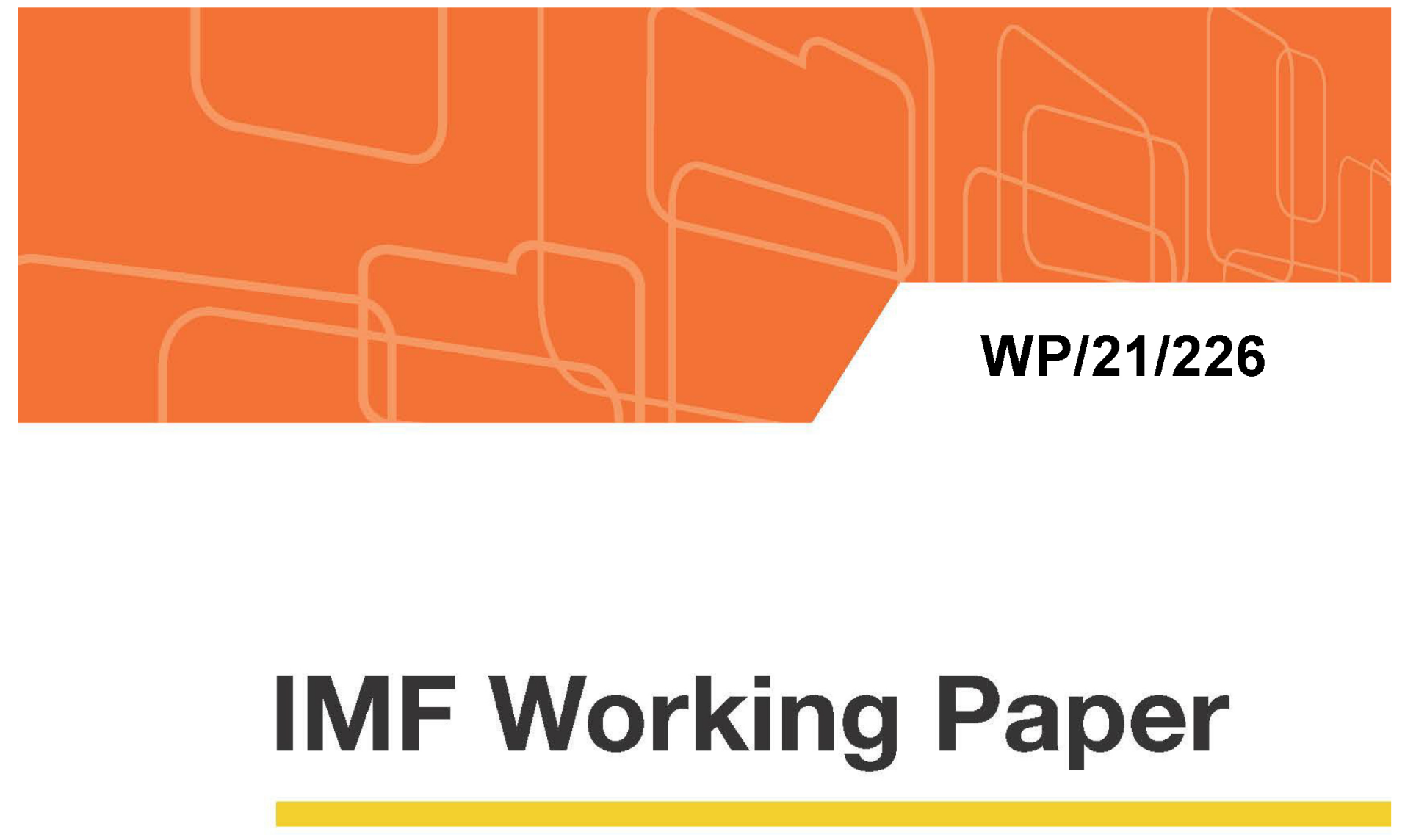

\title{
A Pandemic Forecasting Framework: An Application of Risk Analysis
}

by Allan Gloe Dizioli, Daniel Rivera Greenwood and Aneta Radzikowski

IMF Working Papers describe research in progress by the author(s) and are published to elicit comments and to encourage debate. The views expressed in IMF Working Papers are those of the author(s) and do not necessarily represent the views of the Federal Reserve System and the IMF, its Executive Board, or IMF management. 


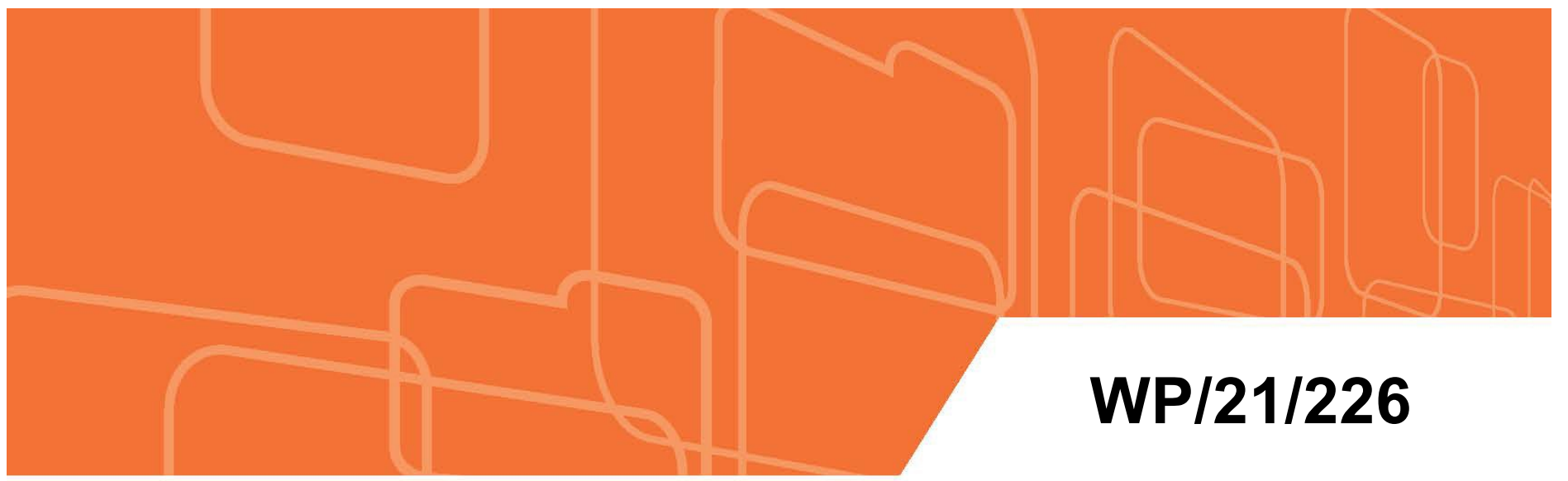

\section{IMF Working Paper}

\section{A Pandemic Forecasting Framework: An Application of Risk Analysis}

by Allan Gloe Dizioli, Daniel Rivera Greenwood and Aneta Radzikowski

IMF Working Papers describe research in progress by the author(s) and are published to elicit comments and to encourage debate. The views expressed in IMF Working Papers are those of the author(s) and do not necessarily represent the views of the Federal Reserve System and the IMF, its Executive Board, or IMF management.

I N T E R N A T I O N A L M O N E T A R Y F U N D 


\title{
IMF Working Paper
}

Research Department

\author{
A Pandemic Forecasting Framework: An Application of Risk Analysis \\ Prepared by Allan Gloe Dizioli, Daniel Rivera Greenwood and Aneta Radzikowski* \\ Authorized for distribution by Benjamin Hunt
}

August 2021

\begin{abstract}
IMF Working Papers describe research in progress by the author(s) and are published to elicit comments and to encourage debate. The views expressed in IMF Working Papers are those of the author(s) and do not necessarily represent the views of the IMF, its Executive Board, or IMF management.
\end{abstract}

\begin{abstract}
This paper introduces a simple, frequently and easily updated, close to the data epidemiological model that has been used for near-term forecast and policy analysis. We provide several practical examples of how the model has been used. We explain the epidemic development in the UK, the USA and Brazil through the model lens. Moreover, we show how our model would have predicted that a super infectious variant, such as the delta, would spread and argue that current vaccination levels in many countries are not enough to curb other waves of infections in the future. Finally, we briefly discuss the importance of how to model re-infections in epidemiological models.

JEL Classification Numbers: E17, D62, I12, I14, J22

Keywords: COVID-19, epidemiology modelling, vaccines impact, virus variants and testing Author’s E-Mail Addresses: adizioli@imf.org; DRiveraGreenwood@imf.org; aradzikowski@imf.org
\end{abstract}

\footnotetext{
*Allan Dizioli, Daniel Rivera Greenwood, and Aneta Radzikowski are, respectively, senior economist and research officers in the IMF Research Department. We are grateful to Benjamin Hunt and Rafael Portillo for us eful comments. All remaining errors are our own.
} 


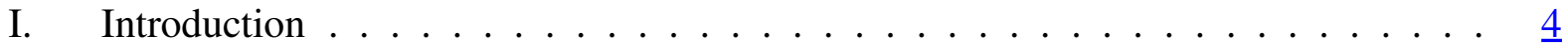

II. Literature Review . . . . . . . . . . . . . . . . . $\underline{6}$

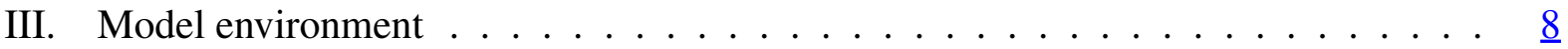

IV. Data and Model Calibration . . . . . . . . . . . . . . . 12

A. Google Mobility . . . . . . . . . . . . . . . . . . 12

B. Our World in Data . . . . . . . . . . . . . . . . . . . 12

C. Vaccination Data ................... . 13

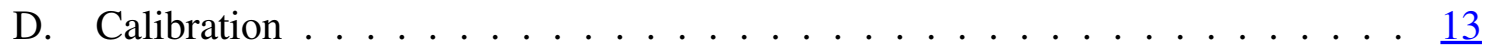

V. Model simulation results . . . . . . . . . . . . . . . . 15

A. UK's model: The importance of early vaccination . . . . . . . . . . 15

B. USA's model: Model explanation of deaths dynamic . . . . . . . . . . . 19

C. Brazil's model and the importance of re-infections . . . . . . . . . . . 22

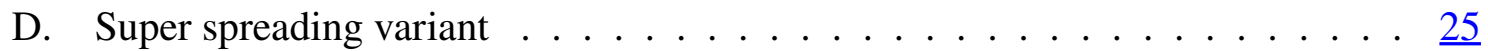

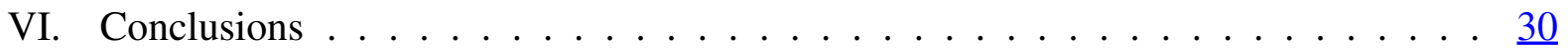

References ........................

Tables

1. Calibrated Parameters . . . . . . . . . . . . . . . 14

Figures

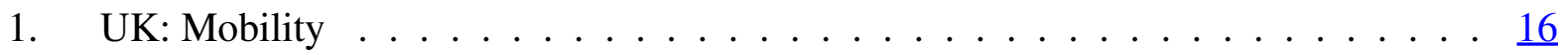

2. UK: Deaths Under Different Infectiousness Assumptions . . . . . . . . . . . 17

3. UK: Infections Under Different Infectiousness Assumptions . . . . . . . . . . 18

4. UK: Infections by Groups . . . . . . . . . . . . . . . . . . . . . 18

5. UK: Deaths Under Different Vaccination Assumptions . . . . . . . . . . 19

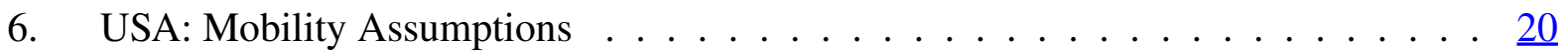

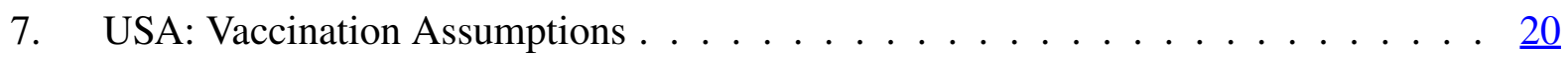

8. USA: Projected Daily Deaths . . . . . . . . . . . . . . . 21 
9. Brazil: Mobility Assumptions . . . . . . . . . . . . . . . . . . 22

10. Brazil: Vaccination Path . . . . . . . . . . . . . . . . 23

11. Brazil: Deaths, Projected and Actual . . . . . . . . . . . . . . 24

12. Elasticity of Domestic Demand to Google Mobility . . . . . . . . . . . . 26 26

13. The Seasonality in Mobility Could Bias the Relationship With Output . . . . . . 26

14. The Unequal Vaccine Rollout Remains a Major Issue in Most of the Developing

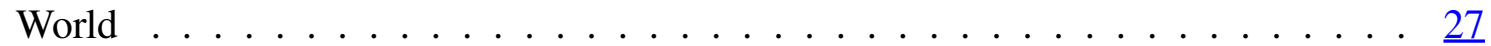

15. Vaccine Hesitancy Will Play Critical Role in the Next Few Months . . . . . . . . 28

16. India Could be Severely Affected by a More Infectious Virus Strain . . . . . . . . 28

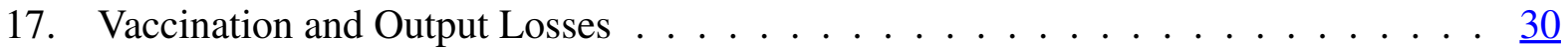

18. Emerging Market Economies Could Suffer Large Output Losses . . . . . . . . . $\underline{31}$ 


\section{INTRODUCTION}

COVID-19 infections and efforts to contain them have driven most of the discussion about the macroeconomic outlook in the last year and a half. While governments mandated lockdowns and stay-at-home orders were enacted to prevent health system collapses, we have also seen voluntary social distancing by agents trying to minimize their virus exposure. All of this has had important economic implications, with contact-intensive sectors experiencing sharp downturns.

Meanwhile, when the pandemic seemed to be under control in late 2020, new more infectious virus variants started to emerge. The alpha variant was the first to spread and it is believed to be around 50 percent more infectious than the original COVID-19 virus. Later on, the beta, gamma and more recently the delta variants were identified, with the delta variant believed to be substantially more infectious than the alpha one. Finally, a massive vaccination campaign was initiated in late 2020 in a few countries and has spread globally more recently. While for many months vaccine supply constraints were the most important factor limiting vaccination, vaccine hesitancy has recently started to bind in a couple of advanced economies. Moreover, countries have adopted distinct vaccination strategies and have used different vaccines with diverse effectiveness against virus strains.

This paper presents a simple epidemiological model to make sense of all this information and to explain the epidemic dynamics in different countries. The major goal of the model is to produce short-term epidemic forecasts, given projected vaccination rates, agents' mobility and the emergence of new more infectious variants. However, the model can also be used to assess the impact of non-pharmaceutical interventions, the impact of vaccination campaigns and the quantification of risk scenarios. The model's link to economic activity comes from the derivation of a mobility path that is consistent with a controlled path for the pandemic. Subsequently, other outside models can be used to translate this mobility path into economic forecasts.

In the results section, we provide several examples of how the model has been used at the International Monetary Fund (IMF). We explain the epidemic development in the UK, show the importance of the alpha variant in explaining infections, and show the impact of their early vaccination campaign. Moreover, we show how our model predicts that a super infectious variant, inspired on the delta variant, would spread, including among vaccinated people. Similarly, we show some of these features in a model calibrated to the USA. In particular, our sim- 
ulations indicate that the current vaccination levels in the USA are not high enough to curb another wave of infections in the context of a highly transmissible virus strain. Finally, we use a model calibrated for Brazil to show how important the assumption about re-infections is when forecasting deaths and infections going forward.

While this first version of the model does not directly link mobility to economic outcomes, we provide an example of how a simple model connecting mobility to domestic demand can be linked to our epidemiological model to produce the economic quantification of a new virus variant. In the context of a 50 percent more infectious variant that hits all the countries in the world simultaneously, global growth in 2021 and 2022 is more than 0.8 percentage points weaker compared to a scenario where this variant did not emerge. Given current lower levels of vaccination, emerging and developing economies are more negatively affected in growth terms with GDP growth below baseline by roughly 1 percentage point in 2021 and 2022 . Meanwhile, vaccine hesitancy and global spillovers cost advanced economies around $3 / 4$ percentage points in GDP growth in 2021 and 2022. By 2025, global output is still roughly $1 / 2$ percent below baseline, and the cumulative loss by 2025 is just under $4^{1} / 2$ trillion U.S. dollars.

Our results are certainly relevant from a policy perspective. We are able to measure how public policies such as vaccination of different groups and non-pharmaceutical interventions can affect the pandemic. Moreover, the short-term forecasts can be used as a tool to plan the implementation of those policies. We acknowledge that there is large parameter uncertainty and some facts are still not very well established, for example how reinfections actually happen. In order to deal with these uncertainties, we show how the model predictions would change in case some of the model parameters or modelling choices are different. In particular, in section V.A we show how the virus dynamic would change if a variant is actually twice more infectious than initially estimated, and in section V.C we do a similar analysis with different ways of modelling reinfections. As the results in these sections show, the model implications can be dramatically different given the model's non-linearity. Thus, the model needs to be frequently updated to be sure some elements are correctly specified.

The paper is divided into 6 sections. Section 2 discusses the literature on COVID-19 and some other simple models. Section 3 presents our theoretical model. Section 4 describes the data and model calibration. Section 5 shows country applications and other quantitative global-macro-model based results while Section 6 concludes the paper. 


\section{Literature Review}

The literature on COVID-19 has grown fast and at this stage is extensive. We will not cover all the papers on the topic, but briefly discuss some of the papers most similar to our work. Most of the models used in the epidemiological economics literature are derivations from the SIR, SEIRD or SIRD models, which were first introduced by Kermack and McKendrick (1927). The main feature of these models is to divide the population in states for example, $S$, I, E, R and D (Susceptible, Infectious, Exposed, Recovered or Dead), and people transition between states following laws of motion that are guided by virus specific characteristics. Early on in the pandemic, Atkeson (2020) used a simple SIR model to compare scenarios regarding social distancing and the progression of COVID-19. He modeled social distancing as an exponential average of two states. In our paper, we further decompose behavior between mobility and other measures to mitigate the virus, such as social distancing, wearing masks, etc. The functional form of our infectiousness dynamic was inspired by Fernández-Villaverde and Jones (2020). They use a SIRD model with a time-varying contact rate to capture behavioral and policy-related changes in social distancing. We extend the work of Fernández-Villaverde and Jones (2020) and include a decomposition of infectiousness between the number of substantive contacts that someone has and how infectious the virus is. We also introduce vaccinations and new variants as factors that can change the pandemics dynamic.

Eichenbaum, Rebelo, and Trabandt (2020a) pioneered in presenting a model exploring the interaction between economic decisions and pandemics. The model implies a cut back on consumption and work as people look to minimize the probability of infection. Extending their initial framework, Eichenbaum, Rebelo, and Trabandt (2020b) incorporate viral testing to study the impact of testing, quarantining and non-pharmaceutical interventions on health and economic outcomes. Although our model is simpler in the dimension of modelling endogenous behavior, it links mobility and contacts using Google mobility data and enriches the dynamics with the introduction of new variants and re-infections. This way, our model does a better job in explaining the dynamics observed in the current pandemic.

Bakker and Goncalves (2021) also use a variation of a SEIR model to investigate the effect of public policies on transmission. They use their simple model to show that higher stringency

policies lowered mobility and infections in Latin America, but the results were better in countries with better government effectiveness. Our model extend the basic SEIR model in several dimensions, allowing us to respond to questions on vaccinations, re-infections and new variants that Bakker and Goncalves (2021) did not explore. 
Berger and others (2020) is another example of using a modified SEIR model to study public policies. Their model includes testing (serological and virological), asymptomatic individuals, imperfect information, and a behavioral response. Their paper finds that testing and reopening can slacken the output-mortality trade-off that features in many economic models of pandemics. Meanwhile we use actual mobility data to match our model with the data. Berger and others (2020) has a reduced form equation to model behavior and uses other parameters to fit the model to the data. Most of the papers mentioned, like ours, rely on calibration methods to match the pandemic. One of the few exceptions is Bognanni and others (2020), that estimate a spatial, micro-founded model of the joint evolution of economic variables and the spread of an epidemic and find that mitigation measures that reduce viral transmission (e.g., mask-wearing) both reduce the virus's spread and increase economic activity. This channel is also present in the theoretical model described in the next section.

Our paper provides the theoretical background for the epidemiological model used by DablaNorris and others (2021). They investigate the determinants of vaccine hesitancy and use an older version of our model to assess the effects of vaccine hesitancy on the number of COVID-19 cases and deaths. They show that the effects of vaccination on deaths is exponential over time and defend strategies to speed up vaccination and lower vaccine hesitancy.

On the topic of using epidemiology models to study the impact of vaccination, Moore and others (2021) use an age-structured mathematical model of SARS-CoV-2 transmission to test which group of people should be the first to receive the vaccine to reduce mortality and healthcare demands. They conclude that vaccinating the most elderly and vulnerable first has the greatest impact on deaths. Our theoretical model could also be used to conduct this exercise, as we also model the vulnerable group separately. Differently from our paper, they do not take people's behavior as a major factor in transmission. We also examine the role of testing and quarantining, seasonal factors and new virus variants.

Similar to what we do in one of our result sections, MacIntyre, Costantino, and Trent (2020) try to use a model to measure the impact of vaccinations and propose a strategy for vaccine implementation in Australia. Different from our paper, they do no consider that agents change their behavior over time, affecting the epidemic dynamics.

On the empirical role of vaccinations, Ganslmeier and others (2021) try to measure the effect of vaccination on new cases and macroeconomic activity indicators. Differently from our paper, they empirically link vaccination to economic activity directly, while our main focus is on the impact on potential mobility. They also conclude that a 10 -percentage point increase 
in the share of the vaccinated with at least one dose reduces daily new cases per capita after 21 days by 0.1 percentage point. Our model predicts that this effect is non-linear, and its marginal effect increases with a higher fraction of the population being vaccinated.

\section{Model ENVIRONMENT}

Extending the standard SEIR model, our basic framework divides the population $(\mathrm{N})$ into susceptible $(\mathrm{S})$, infectious $(\mathrm{I})$, recovered $(\mathrm{R})$, quarantined $(\mathrm{Q})$, vaccinated $(\mathrm{V})$, infected vaccinated (Iv) and dead (D) states. Some of these states follow the same pattern as a SEIR model. So, in our daily framework, there is a matching of susceptible and infected populations that result in $\beta_{t} S_{i, t}\left(I_{i, t}+I_{j, t}+I v_{i, t}+I v_{j, t}\right)$ infections, Where $\beta_{t}$ is an endogenous rate of infection that depends on behavioral patterns, mobility and a seasonal component. Its functional form follows:

$$
\beta_{t}=n_{t} i n f_{t} \mu_{t}
$$

where $n_{t}$ is the average number of substantive contacts with other people per day (affected by lockdowns and voluntary social distancing), in $f_{t}$ is the probability of infection conditional on having a substantive contact (which can be reduced by wearing masks, keeping distance, washing hands, etc.), and $\mu_{t}$ is a parameter that adjusts the scale and controls for seasonal differences in the rate of infections.

The number of contacts, $n_{t}$ per day is defined as:

$$
\ln \left(n_{t}\right)=\ln \left(n_{0}\right)-\alpha \ln \left(m_{t}\right)
$$

where $n_{0}$ is the initial number of contacts, $\alpha$ is a parameter that we calibrate and $m_{t}$ is relative 7-day average mobility in day $t$, measured in relation to the pre-pandemic baseline. We use google transportation mobility data to proxy the changes in the number of contacts that someone has.

Agents slowly learn about the virus and get better at avoiding infections in a given interaction. For example, agents promote social distancing while communicating, they wash hands and they use masks. In order to capture these behavioral patterns, we follow Fernández-Villaverde 
and Jones (2020) and model this learning process as an exponential average. Thus, the probability of contagion evolves according to:

$$
\inf f_{t}=\beta_{0} e^{-\lambda t}+\beta_{s} \Psi\left(1-e^{-\lambda t}\right)
$$

where $\beta_{0}$ is the initial rate of infection, $\beta_{s}$ is the rate of infection when safety precautions are taken, and $\lambda$ measures the time to make that transition. Finally, the $\Psi$ is introduced to capture new COVID-19 variants. Thus, when a variant is twice more infectious than the original COVID-19 variant, we have $\Psi=2$. Notice that once the new variant starts spreading, it just expands the number of states in our model to include infected agents with the new variant. The different variants co-exist for a small period but eventually almost all new infections would come from the most infectious variant. The larger the value of $\Psi$, the shorter this period is. Once we introduce more than one variant, what matters is the difference in infectiousness between the two most infectious variants. Finally, the seasonal factor, $\mu_{t}$, is modelled as a sinusoidal function that reaches its peak at mid-winter and we estimate its amplitude according to the country being modelled.

Another extension to the SEIR model is the quarantined category. This category is adopted to accommodate both asymptomatic and pre-symptomatic transmissions and to allow for random testing in the model. The difference between people who are infectious and people who are quarantined is that those in quarantine no longer transmit COVID-19 because they do not have contact with others. The sooner these people are taken out of circulation, either because of a positive COVID test, $q_{t}$, or because of the development of symptoms, $\gamma$, the lower the speed of the spread of the virus.

Once in the quarantine state, agents can either recover from the virus and move to the recovered state, $r_{t}$, or they die with a probability $\delta_{t}$. If agents recover, they acquire temporary immunity from the virus until they receive a new shock, $\kappa$, and move back to the susceptible state. The value of $\kappa$ is calibrated so that it takes an average of 6 months for someone to be susceptible again.

We have also introduced the vaccination state to the basic model. We assume that a fraction $\rho$ of the population subgroup is vaccinated daily. COVID-19 infections are still possible and depend on the vaccine's effectiveness, $\omega$. In the model, we allow the vaccines' effectiveness to be different for the distinct COVID-19 variants. Once in the infected vaccinated state, agents eventually recover to be part of the vaccinated state again. This means that we assume that no one dies from a COVID-19 infection after vaccination. 
Other than these population state variables, we also have two distinct groups that differ in terms of their probability of dying after a COVID-19 infection. We name those groups as nonvulnerable and vulnerable, which we define in practice as the population over 65 . While we know that in the real world these two groups do not socialize with the same intensity and interact more within than between groups, we make the simplifying assumption that they only differ in terms of their probability of dying. We acknowledge that this might miss some of the developments occurring within the groups, but we think that the assumption produces a good enough approximation for the purposes of our model.

The differences in mortality between these two groups implies that a country with an older population will have higher average infection mortality rate in our model. The distinction of these two groups also allows us to study the public policy of first vaccinating the vulnerable groups, a strategy widely used at the beginning of the vaccination roll-out, when vaccine supply was the major issue countries were facing.

With these seven states described above, the model evolves according to the following laws of motion:

$$
\begin{array}{ll}
S_{i, t+1}-S_{i, t}=-\beta_{t} S_{i, t}\left(I_{i, t}+I_{j, t}+I v_{i, t}+I v_{j, t}\right)-\rho_{i, t} S_{i, t}+\kappa r_{i, t} & \left(L M_{1}^{i}\right) \\
I_{i, t+1}-I_{i, t}=\beta_{t} S_{i, t}\left(I_{i, t}+I_{j, t}+I v_{i, t}+I v_{j, t}\right)-\gamma \frac{I_{i, t}}{\left(1-q_{t}\right)} & \left(L M_{2}^{i}\right) \\
Q_{i, t+1}-Q_{i, t}=-\theta Q_{i, t}+\gamma \frac{I_{i, t}}{\left(1-q_{t}\right)} & \left(L M_{3}^{i}\right) \\
V a_{i, t+1}-V a_{i, t}=\rho_{i, t} S_{i, t}-\beta_{t} \omega_{t} V a_{i, t}\left(I_{i, t}+I_{j, t}+I v_{i, t}+I v_{j, t}\right)+\theta I v_{i, t} & \left(L M_{4}^{i}\right) \\
I v_{i, t+1}-I v_{i, t}=\beta_{t} \omega_{t} V a_{i, t}\left(I_{i, t}+I_{j, t}+I v_{i, t}+I v_{j, t}\right)-\theta I v_{i, t} & \left(L M_{5}^{i}\right) \\
r_{i, t+1}-r_{i, t}=\theta\left(1-\delta_{t}\right) Q_{i, t}-\kappa r_{i, t} & \left(L M_{7}^{i}\right) \\
d_{i, t+1}-d_{i, t}=\theta \delta_{t} Q_{i, t} & \left(L M_{8}^{i}\right)
\end{array}
$$

where $i, j$ stand for each of the groups (non-vulnerable and vulnerable), and so we have 16 laws of motion in total. The time varying $\beta_{t}$ measures the rate of infection, $\rho$ measures the daily vaccination rates of that specific group, $\gamma$ is the rate at which symptoms develop, $\theta$ is the time it takes to recover from an infection, $\omega$ is how efficient the vaccine is in cutting transmission, and $\delta$ is the probability of death given an infection. The expression $\frac{\gamma_{t}}{\left(1-q_{t}\right)}$ is the rate at which an infected individual discovers that he/she is infected. The reasoning is as follows: once a person is infected, symptoms develop at Poisson rate $\gamma$, which means that the average number of days until the first symptoms appear is $\frac{1}{\gamma_{t}}$. When a fraction $q_{t}$ of the population is tested each day, the average number of days until a person either develops symptoms or re- 
ceives a positive result is $q_{t} 0+\left(1-q_{t}\right) \frac{1}{\gamma_{t}}$ inverting this expression gives the rate at which infections are discovered.

Finally, notice that we do not include the new variants here for the sake of presentation simplicity. However, for every new variant that arrives, there will be two additional states to the system. We can define them as $I_{t}^{n v}$, for the individuals infected by the variant $n v$, and $I v_{t}^{n v}$, for the vaccinated individuals infected by the same variant. For example, when a new variant is included, the susceptible state evolves according to:

$$
S_{i, t+1}-S_{i, t}=-\beta_{t} S_{i, t}\left(I_{i, t}+I_{j, t}\right)-\beta_{t} \Psi_{t} S_{i, t}\left(I_{i, t}^{n v}+I_{j, t}^{n v}\right)-\rho_{i, t} S_{i, t}+\kappa r_{i, t}
$$

Following Dizioli and Pinheiro (2020), we allow the death rate to be dependent on ICU hospital capacity. In particular, we assume that $\delta_{t}$ has the following functional form:

$$
\delta_{t}= \begin{cases}\bar{\delta} & \text { if } \frac{H o_{t}}{I C U_{t}}<0.5 \\ \bar{\delta}+\left(\frac{H o_{t}}{I C U_{t}}\right)^{2} \frac{1}{K} & \text { if } \frac{H o_{t}}{I C U_{t}} \geq 0.5\end{cases}
$$

where $\bar{\delta}$ is the mortality rate in the case where no physical constraint is considered, $H o_{t}$ is the number of hospitalizations, $I C U_{t}$ is the number of ICU beds, and $K$ is a constant used for calibration. For $\bar{\delta}$, we use our benchmark calibration. For the number of hospitalizations, we assume that 20 percent of sick people need to be hospitalized and 5 percent of them would require ICU treatment. For the number of ICU beds, we follow McCarthy (2020). ${ }^{1}$ Finally, we calibrate $K$ so that the probability of dying increases by 20 percent during periods of strain on the health system. We assume that periods of strain on the health system are periods in which the ICU capacity utilization is greater or equal to 50 percent. $^{2}$

Mobility not only has an effect on the pandemic, but also on economic activity. Once people restrain mobility voluntarily or following government rules, they tend to consume less, especially from intensive-contact sectors. Once we model the relationship of mobility to economic outcomes, we can use our model to make economic projections under the different scenarios. While our simple model does not try to make this link between mobility and the economy explicit, Section V.D shows an example of how it can be used in this context.

${ }^{1}$ Information from the National Center for Biology Information, Intensive Care Medicine. ${ }^{2}$ See Odone and others (2020) for our calibration's motivation. 


\section{Data and Model Calibration}

\section{A. Google Mobility}

We use Google Mobility data ${ }^{3}$ to estimate the number of contacts people have per day. Google LLC (2021) Google Mobility Reports show how visits to places, such as grocery stores and parks, are changing in each geographic region. The data is available for the following categories: grocery and pharmacy, parks, transit stations, retail and recreation, residential, and workplaces. In this paper, we use either workplaces or transit stations data, depending which one produces a better fit for a specific country.

The data is shown as deviation from a pre-COVID baseline (the median value for that day of the week during the period Jan 2, 2020-Feb 6, 2020). The changes are calculated using aggregated and anonymized data collected from mobile devices, the same data that is used to show popular times for places in Google Maps. The data is available at the daily frequency, with only a 2-3 days lag. We smooth the data to remove excess volatility in the daily data by using a 7-day moving average, as mobility during weekends and holidays tends to be lower.

We acknowledge the limitations of this data, as it captures data from only a sample of Google users, those who have opted-in to Location History for their Google Account, and does not necessarily represent the whole population of that country. This problem might be particularly worse in developing economies, where the cell phone coverage might not be representative of society.

\section{B. Our World in Data}

We use Our World in Data COVID-19 dataset ${ }^{4}$ to access most epidemiology data. We followed the strategy in Fernández-Villaverde and Jones (2020) and use new daily deaths to calibrate our models. Daily deaths tends to be more reliable than infections data when calibrating models across countries. That is because different countries have different testing capabilities, and several might miss a considerable number of infections, especially the asymptomatic ones. Similarly to the mobility data, we use a 7-day moving average. We also use Our World in data for vaccine distribution.

${ }^{3}$ https://www.google.com/covid19/mobility/

${ }^{4}$ https://github.com/owid/covid-19-data/tree/master/public/data 
The data on deaths is updated daily and comes from COVID-19 Data Repository by the Center for Systems Science and Engineering (CSSE) at Johns Hopkins University. Due to the long reporting process between the death occurrence and its inclusion in statistics, the data may come with a lag, while the vaccination data is collected by the Our World in Data team from official reports.

\section{Vaccination Data}

For our vaccination assumptions, we use the vaccination data from Hannah Ritchie and Roser (2020) for the daily first and second vaccine doses. However, the dataset has only historical values and doesn't provide a breakdown by age. We use our judgment and data from authorities for the projections and to divide the doses into the vulnerable and non-vulnerable populations. For the UK model, we use National Health Service (2021) which provides vaccination by doses and by age for England and apply a similar vaccine distribution for the rest of the countries. For the projections, we assume that vaccinations will continue at the same pace for the younger population, but will reach a peak for people of age 65 and older. For Brazil and the United States models, we also rely on estimates from Hannah Ritchie and Roser (2020) for historical values. For projections, we use the Brazilian government's contracted vaccines schedule and assumed that the coverage would reach levels consistent with surveys on vaccine hesitancy, such as Datafolha in Brazil ${ }^{5}$. For the USA, supply is no longer a concern and the pace of vaccination is mostly determined by demand, so we also cap vaccinations based on willingness-to-take-vaccines surveys. ${ }^{6}$.

\section{Calibration}

We developed models for several countries and some parameters are country specific to capture the evolution of the pandemic in those countries. In this section, we discuss the common parameters in all models, and in the results section we discuss particular country calibrations.

The model is calibrated so that a time period is a day. We assume that it takes an average of two weeks to either recover or die from the infection -that is $\theta+\delta=1 / 14$. The parameter

\footnotetext{
${ }^{5}$ https://www1.folha.uol.com.br/equilibrioesaude/2021/07/adesao-a-vacina-chega-a-94-e-atinge-recorde-nobrasil.html

${ }^{6}$ https://www.ipsos.com/en/global-attitudes-covid-19-vaccine-january-2021
} 
$\gamma$ is calculated so that the incubation period is around 10 days. The re-infection rate shock, $\kappa$ is calculated so that a re-infection occurs on average after 6 months (a number also used by the CDC). We use Ward and others (2020) to base our calculation of the relative probability of death (once infected) of the vulnerable, $\delta_{v u, t}$ and the non-vulnerable, $\delta_{n v, t}{ }^{7}$. We mostly use Census data to calculate population shares $s_{v u}$ and $s_{n v}$, where we define the population aged 65 and over as the vulnerable population.

The pre-pandemic number of daily contacts is calibrated using results from the American Community Survey (ACS), and relative mobility is available through Googles Community Mobility Reports. The parameters $\alpha, \beta_{0}, \beta_{s}, \lambda, \Psi$ are country specific and are calibrated or estimated to track each country's 7-day moving average of new deaths. Finally, the vaccine efficacy parameter is calculated by country depending on which vaccine the country has been using. Table 1 summarizes the parameter choices for the models discussed in this paper.

Table 1. Calibrated Parameters

\begin{tabular}{|c|c|c|c|c|}
\hline Parameter & $\mathbf{U K}$ & US & Brazil & Description \\
\hline$\beta_{0}$ & 0.1700 & 0.1445 & 0.1500 & The initial rate of infection \\
\hline$\beta_{s}$ & 0.07650 & 0.07624 & 0.06700 & $\begin{array}{l}\text { The rate of infection when safety precau- } \\
\text { tions are taken }\end{array}$ \\
\hline$\lambda$ & 0.028 & 0.035 & 0.028 & $\begin{array}{l}\text { Parameter guiding the speed of transition } \\
\text { from } \beta_{0} \text { to } \beta_{s}\end{array}$ \\
\hline$\gamma$ & 0.17 & 0.17 & 0.17 & $\begin{array}{l}\text { Parameter guiding how long it takes to de- } \\
\text { velop symptoms }\end{array}$ \\
\hline$\theta$ & 0.12 & 0.12 & 0.12 & $\begin{array}{l}\text { The time it takes to recover from an infec- } \\
\text { tion }\end{array}$ \\
\hline$\mu_{t}$ & 1.490 & 1.330 & 1.327 & $\begin{array}{l}\text { Adjusts the scale and controls for seasonal } \\
\text { differences in the rate of infections }\end{array}$ \\
\hline$v i$ & 3.0 & 3.0 & 2.5 & $\begin{array}{l}\text { Reduction in infectiousness after vaccine. A } \\
\text { number } 3 \text { means } 67 \text { percent less infectious }\end{array}$ \\
\hline$\delta_{v u, t}$ & 0.024730 & 0.021277 & 0.024730 & $\begin{array}{l}\text { The relative probability of death of the vul- } \\
\text { nerable }\end{array}$ \\
\hline$\delta_{n v, t}$ & 0.000690 & 0.000709 & 0.000690 & $\begin{array}{l}\text { The relative probability of death of the non- } \\
\text { vulnerable }\end{array}$ \\
\hline$s_{v u}$ & 0.180 & 0.165 & 0.150 & The share of the vulnerable population \\
\hline$s_{n v}$ & 0.820 & 0.835 & 0.850 & The share of the non-vulnerable population \\
\hline
\end{tabular}

${ }^{7}$ We scaled it down a bit to improve the models' fit. 


\section{Model Simulation Results}

\section{A. UK's model: The importance of early vaccination}

This section uses our model to show how the UK's fast vaccine rollout averted an even larger infection wave following the emergence of the alpha variant late last year. According to our model simulations, which start in July 19th, 2021, deaths could have been more than twice as high at the peak of deaths in the first quarter of 2021 if mobility had been the same but no vaccines had been deployed. Moreover, the delta variant would have produced another wave of deaths around July 2021.

Before we discuss the model results in greater detail, let's discuss some of the UK model calibration choices. We calibrated the initial probability of getting infected $\left(\beta_{0}\right)$ at 17 percent, and that is lowered to around 7.7 percent after the population starts taking precautions (such as, wearing masks, social distancing, etc $\left.\left(\beta_{S}\right)\right)$. The parameter guiding the speed of transition to lower infection probability $(\lambda)$ is estimated to be 0.028 . Symptoms develop on average after 5 days $(\gamma)$, while the time it takes to recover from an infection $(\theta)$ is 9 days. Using Ward and others (2020) and our judgment, we have estimated that the probability of dying once infected with COVID-19 $\left(\delta_{n v, t}\right)$ is 0.07 percent for people who are below 65 and 2.47 percent for people 65 and older $\left(\delta_{v u, t}\right)$. The parameter guiding re-infections was set so that on average someone can get reinfected 200 days after the previous infection.

A core part of our scenario is to estimate how mobility affects contacts and to make mobility projections going forward. We use the Google Mobility to workplace to estimate the historical values of the daily contacts until July 12, 2021. For the future contacts, we assume that contacts will gradually increase over time, as more people get vaccinated, and lockdowns and other restrictions are gradually lifted. In particular, contacts reach pre-pandemic level at the end of April 2022, when mobility is back to normal (Fig 1 shows our mobility assumptions).

The vaccination campaign in the UK was successful and reached a large part of the population. However, a large fraction of the population remains vulnerable and we do not predict them to be completely covered. Between December 8, 2020 and July 18, 2021, nearly 70 percent of the population received the first dose and 54 percent were fully vaccinated, but in the United Kingdom, like in many other countries, the first vaccination phase was mainly focused 
Figure 1. UK: Mobility

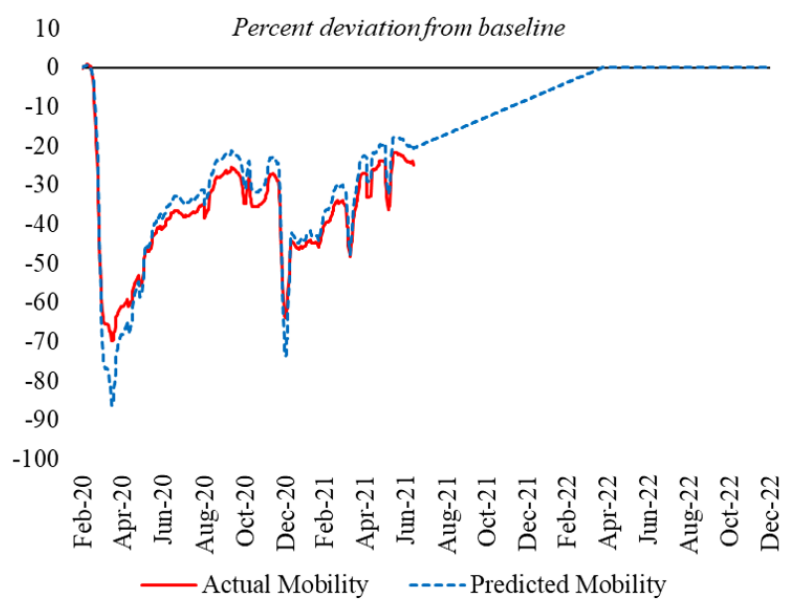

on the vulnerable groups. In our model, the UK concludes its vaccination campaign on August 23, 2021, when about 65 percent of the population below 65 has received the first dose and 55 percent are fully vaccinated, and 95 percent of the vulnerable population has received the first dose and 90 percent both doses. The willingness to vaccinate might increase in the near future, but given current trends, we believe that it is likely that the coverage will be lower than initially anticipated and that some people will not get fully vaccinated.

The model uses vaccination rates of fully vaccinated individuals. However, because of the UKs policy to delay the distribution of the second dose for up to 12 weeks, we have assigned 75 percent protection against the virus after receiving the first dose and remaining 25 percent after the second dose. As the delta variant seems considerably more infectious than the alpha variant, we introduced this third variant at the beginning of June 2021 for the simulations. There is also uncertainty of when the delta variant started to circulate in the UK, with some evidence that started to circulate as early as March ${ }^{8}$. If the variant emerged earlier in the UK, the dynamic can also be different than the one stated here. As our central scenario, we assume it to be 50 percent more infectious than the alpha variant. However, since we are still uncertain about this parameter ${ }^{9}$, we simulate the model with different infectiousness values to check how robust the forecast would be when we increase infectiousness considerably. Fig 2 shows the simulation results when we assume a 50 percent or an 100 percent increase in infectiousness when compared to the alpha variant.

\footnotetext{
${ }^{8} \mathrm{https} / / /$ nextstrain.org/ncov/open/europe

${ }^{9}$ See https://www.yalemedicine.org/news/5-things-to-know-delta-variant-covid for a discussion of the infectiousness of the delta variant.
} 
If the third variant was in fact 100 percent more infectious, instead of our 50 percent baseline, it would result in a 12.5 percent increase in the number of deaths recorded through the end of 2022, as shown in fig 2 . The structure in our model will allow us to independently assess which of these values is more likely given ongoing and future developments in infections and deaths. For example, if the delta variant is indeed 100 percent more infectious than the alpha variant, we should have seen a large spike in infections starting mid-July 2021 and peaking at the beginning of September 2021, which should result in an increase in the number of deaths. While, we have recently seen a small uptick in the number of deaths (fig 3 ) it may be temporary. It is therefore too early to assess what is the actual infectiousness of the delta variant.

Figure 2. UK: Deaths Under Different Infectiousness Assumptions

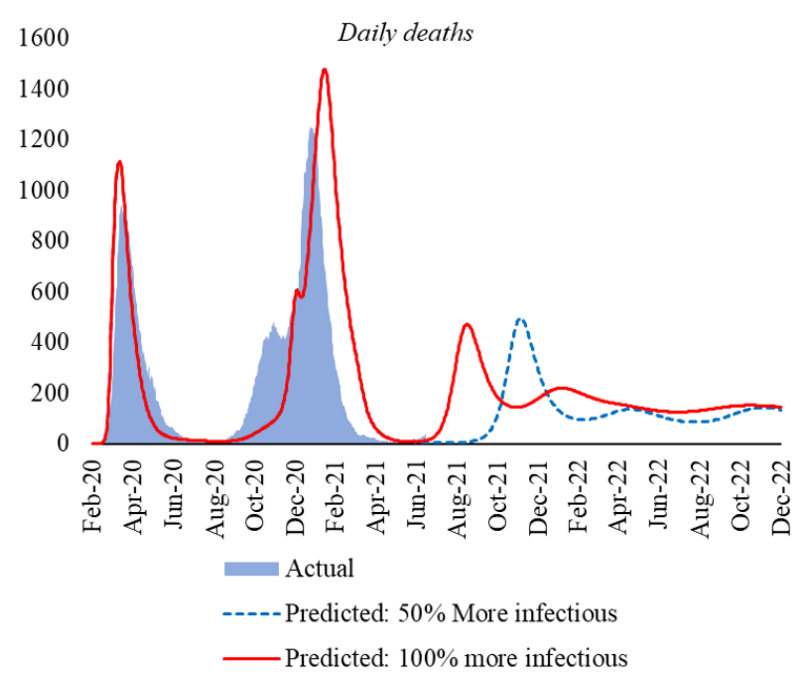

We have also looked at how many new infections are coming from the vaccinated people and how many from non-vaccinated (fig 4). In our projections about 35 percent of total infections come from vaccinated people during the second half of 2021, a share that increases to almost 50 percent during the remainder of the forecast horizon. Our findings show a slightly smaller number than the ZOE COVID Study run by King's College, which states that almost 47 percent of the newly infected people where those who received at least one dose of the vaccine. ${ }^{10}$ The difference is likely coming from our model's structure which only uses the vaccination rates of fully vaccinated individuals. Moreover, we are implicitly assuming that vaccinated and non-vaccinated people behave the same way. If vaccinated people were less

${ }^{10}$ https://www.businessinsider.com/uk-half-covid-19-cases-had-vaccine-study-zoe-delta-2021-7 
Figure 3. UK: Infections Under Different Infectiousness Assumptions

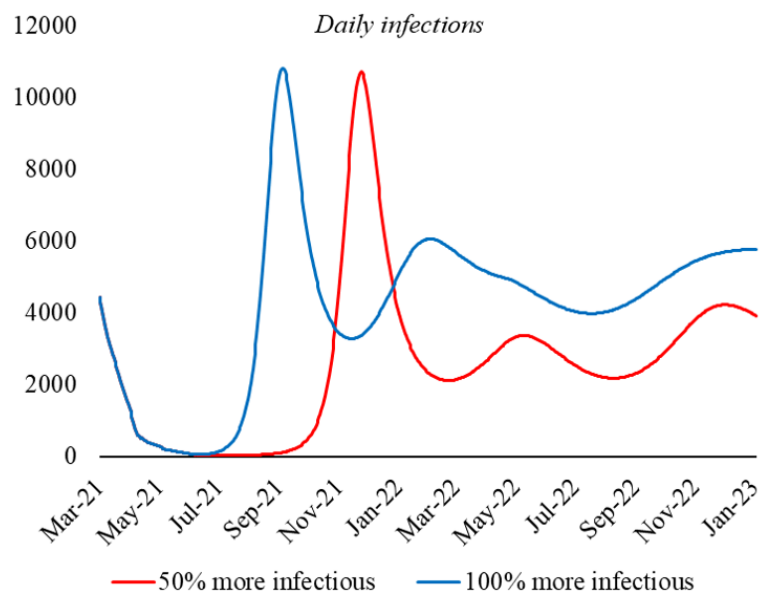

careful around other people, and did things such as not wearing masks or engaging in other riskier behavior, that could raise the share of vaccinated people that get infected. However, our model has done a decent job in tracking those relative infections so far.

Figure 4. UK: Infections by Groups

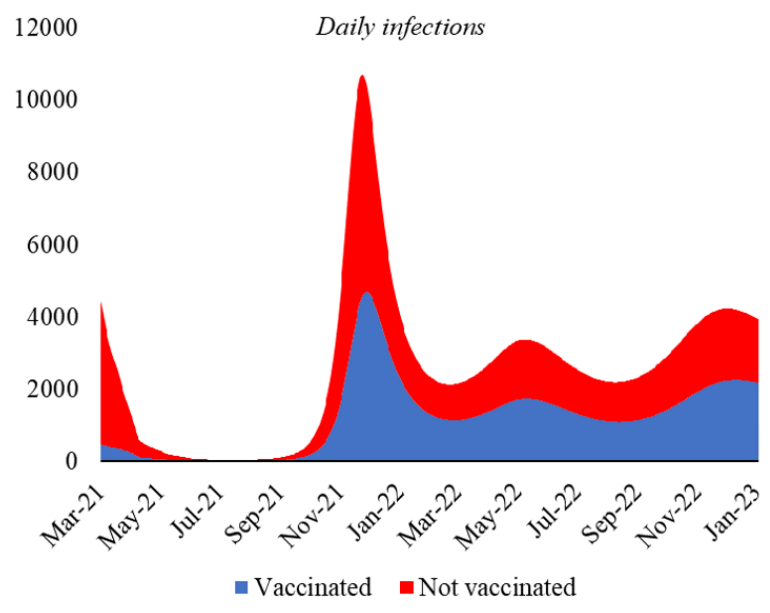

Finally, to illustrate the importance of the vaccination campaign in the UK so far, we present two scenarios: one scenario with our baseline vaccination assumptions, and a second scenario where no one ever got vaccinated. The results are shown in fig 5 . We find that the scenario with no vaccines would have resulted in an increase in the number of deaths by about 
78 percent by the end of 2022, illustrating the number of lives potentially saved by the introduction of the vaccines. One can also see that the number of deaths in 2022 would be similar in the two scenarios. The reason is that in the scenario without the vaccine many more people would get immunity through previous COVID-19 infection. However, the cost of this immunity would have been many more deaths in 2021 .

Figure 5. UK: Deaths Under Different Vaccination Assumptions

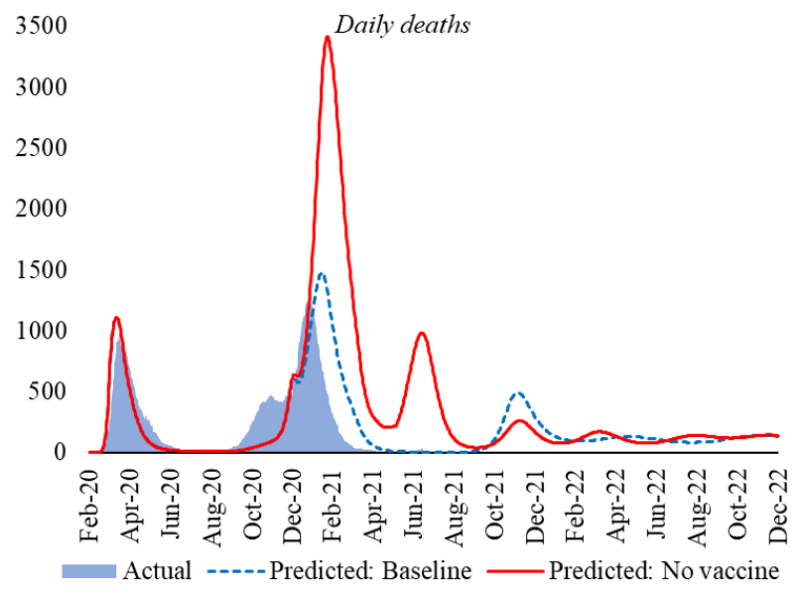

\section{B. USA's model: Model explanation of deaths dynamic}

The model calibrated for the United States can help illustrate three three main factors driving death dynamics: mobility, vaccination pace, and the arrival of new variants with a higher transmissibility. In terms of mobility, we assume people remain cautious until infections drop considerably, either through immunization or immunity through infection. The model forecasts always start on July 19th, 2021. The mobility assumptions are also based on COVID19 Google Mobility Reports (workplace mobility) and we assume a gradual recovery to preCOVID levels. Recent releases highlight that while workplace mobility has not increased as expected - likely due to flexible work arrangements and a reluctance to return to office settings - mobility for retail and recreation has recovered faster than expected, which offsets some of this effect. 
Figure 6. USA: Mobility Assumptions

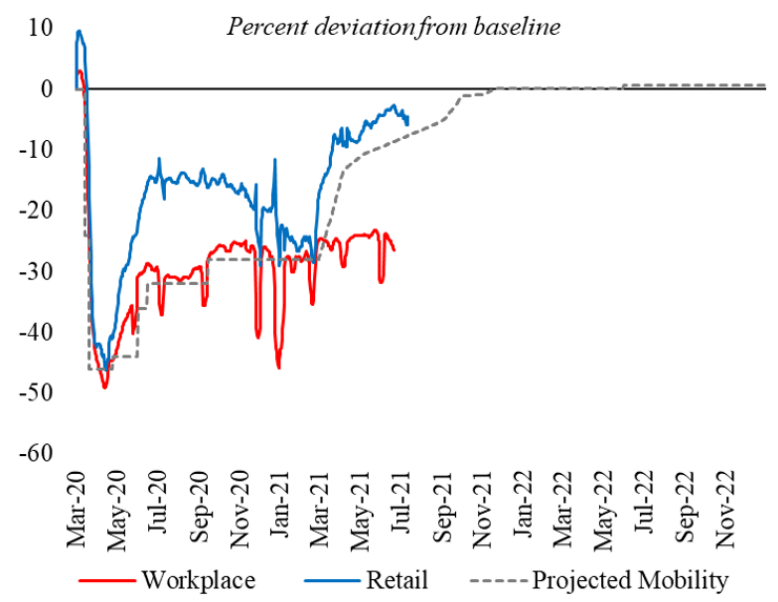

Vaccinations in the United States started fast but have slowed recently, with a relatively high hesitancy. The model assumes that around 60 percent of the population will be vaccinated by the end of 2022. The vaccination campaign focused on individuals at risk first, so vulnerable groups received the vaccine doses early in the campaign, with vaccinations for the general population following afterwards.

Figure 7. USA: Vaccination Assumptions

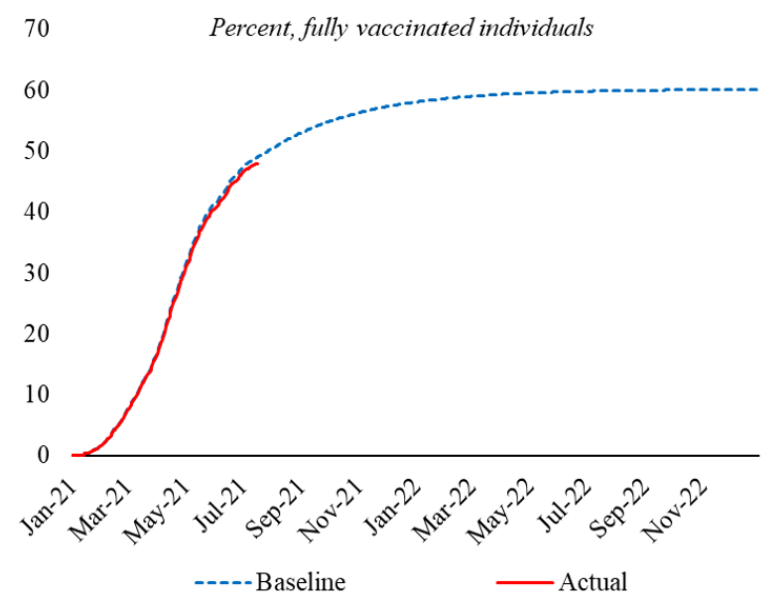

A second variant is introduced in October 2020, estimated to to be 33 percent more contagious than the original variant to match the observed data, while a third variant introduced 
in June 2021 is two times more contagious. We also assume that reinfection is possible after 200 days from vaccination (2nd dose of 2 dose vaccine, or single dose shot) or after original infection date if recovered.

Actual data and projections for the United States show 3 distinct waves of the pandemic. The first, in early-to-mid-2020 was driven by the initial variant and lack of knowledge about the virus, and it was offset by subsequent lower mobility, from lockdowns and voluntary social distancing. As more people used masks and practiced social distancing, the probability of infection given an interaction was reduced and this is confirmed that in our model estimation. The second wave, starting in late 2020 and continuing through the first quarter of 2021 was initially driven by the recovery in mobility and prolonged by the emergence of the alpha variant. The wave declined rapidly as progress in vaccinations increased immunity. The third wave is expected to peak in November 2021; as the vaccination pace in our baseline simulation is not enough to offset the increased contagiousness of the third variant.

Figure 8. USA: Projected Daily Deaths

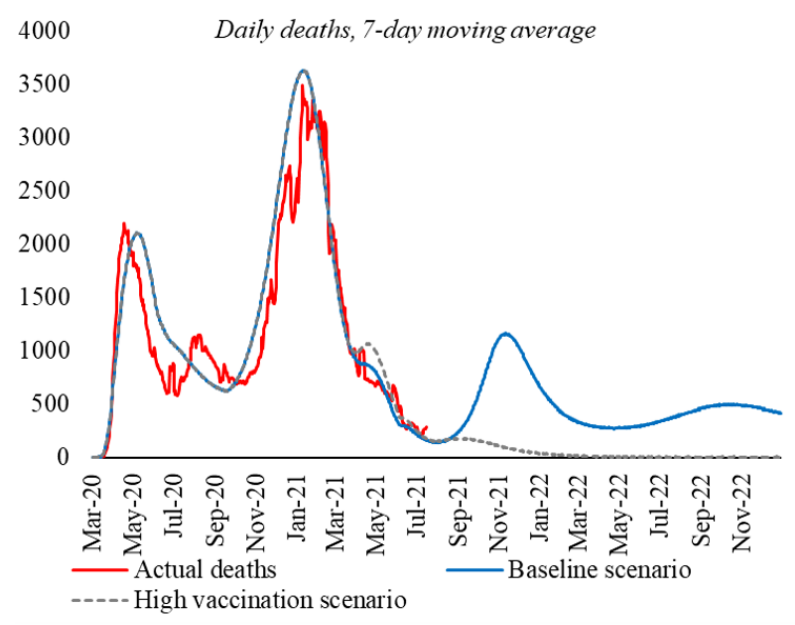

We also provide additional scenarios to show what it would take for the USA to completely avert another wave of deaths. According to our simulations, if the vaccination rate increases twofold relative to the baseline (starting August 1st, 2021), which means an increase in daily average full vaccinations from around 260,000 to 520,000 people, then deaths by the end of 2022 would be reduced by approximately 8 percent. Quadrupling vaccine administration during the same time frame would reduce deaths by 24.2 percent. While large, both scenarios 
are possible, given available vaccine stocks and distribution. The USA was vaccinating over 3 million people daily back in April.

\section{Brazil's model and the importance of re-infections}

We use the model calibrated for Brazil to illustrate the importance of modeling re-infections for the pandemics forecast in the context of a more infectious variant. In particular, we show that if re-infections are not possible, the pandemics would be mostly controlled going forward. Conversely, if re-infections are a possibility and depending on how it is modelled, we can expect another wave of infections by year end.

\section{(a) Building a scenario}

Figure 9. Brazil: Mobility Assumptions

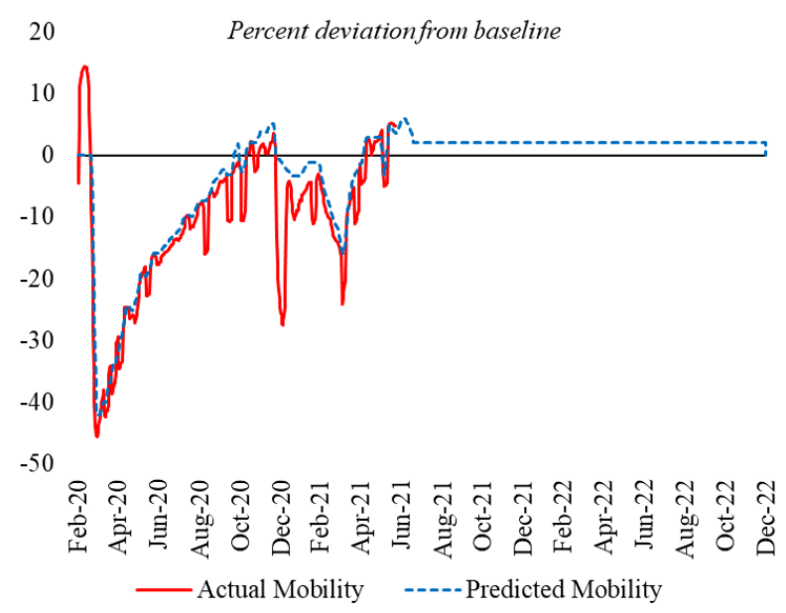

One critical element in building the pandemics forecast is the assumption on how people will behave going forward. Our mobility assumptions are grounded on the COVID-19 Google Mobility Reports (specifically the workplace measure). According to this measure, mobility in Brazil has surprised on the upside and it was above pre-pandemic levels when we start the model forecasts by July 19th, 2021. We believe that the current mobility path is a reaction to optimism to the on-going vaccination campaign and that the mobility path will gradually return to pre-pandemic levels going forward. Moreover, we assume that people would remain 
careful in their daily interactions until infections drop considerably. That is, people will continue to use masks and take other precautions when interacting. As in other countries, Brazil initially vaccinated vulnerable groups.

As this group was fully vaccinated, the general population followed. The vaccination path in the model incorporates actual vaccination rates for fully vaccinated individuals (15.4 percent of the population on July 14th, 2021). As for projections, the model assumes that Brazil will continue to vaccinate its population until 94 percent of the population is fully vaccinated. This target was based on a COVID-19 vaccination willingness poll by Datafolha in Brazil (July 2021) ${ }^{11}$, which along with another Ipsos/World Economic Forum (February 2021) ${ }^{12}$ pool highlight the population's high willingness to get vaccinated.

Figure 10. Brazil: Vaccination Path

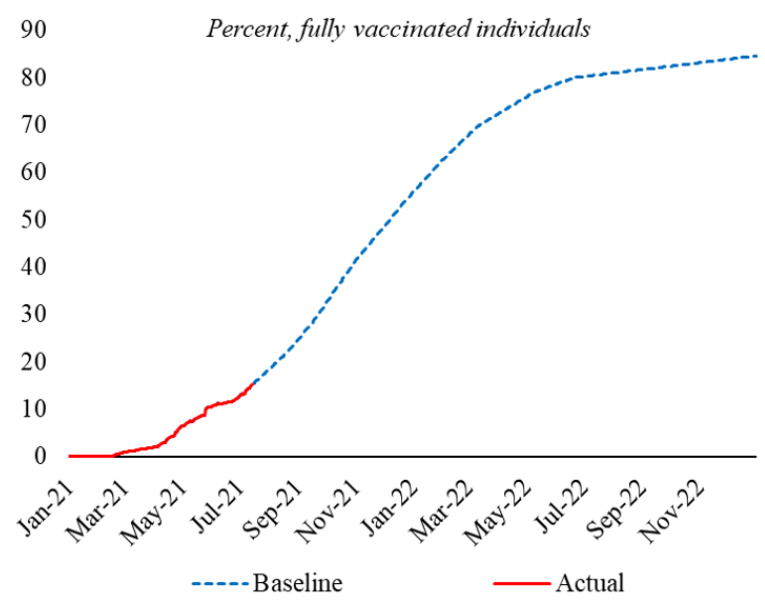

In our scenarios, 75 percent of the population will be fully vaccinated by the end of February 2022, with 75 percent of vulnerable individuals fully vaccinated by July 7 th, 2021. We also include a second COVID variant (P1), more contagious than the original variant, which is introduced in late winter. To illustrate the role of re-infections, we introduce a third variant in late May 2021, even more infectious than the P1 variant. This third variant is 2.8 times more contagious than the original COVID-19 strain.

${ }^{11}$ https://www1.folha.uol.com.br/equilibrioesaude/2021/07/ adesao-a-vacina-chega-a-94-e-atinge-recorde-no-brasil.html ${ }^{12}$ Which finds 88 percent of the population willing to obtain a vaccine. https://www.ipsos.com/en/ global-attitudes-covid-19-vaccine-january-2021 


\section{(b) Model results with and without re-infections when a third wave is modelled}

When re-infections are not allowed, the P1 variant has to be around 57 percent more contagious than the original variant so as to replicate the observed pandemic path ${ }^{13}$. In this model, when the third wave hits the country in June 2021, the combined population immunity gained through either the recovery from previous infections or from the projected vaccination path is enough to avert another wave of infections in the second half of 2021 and early 2022. (Fig 11).

\section{Figure 11. Brazil: Deaths, Projected and Actual}

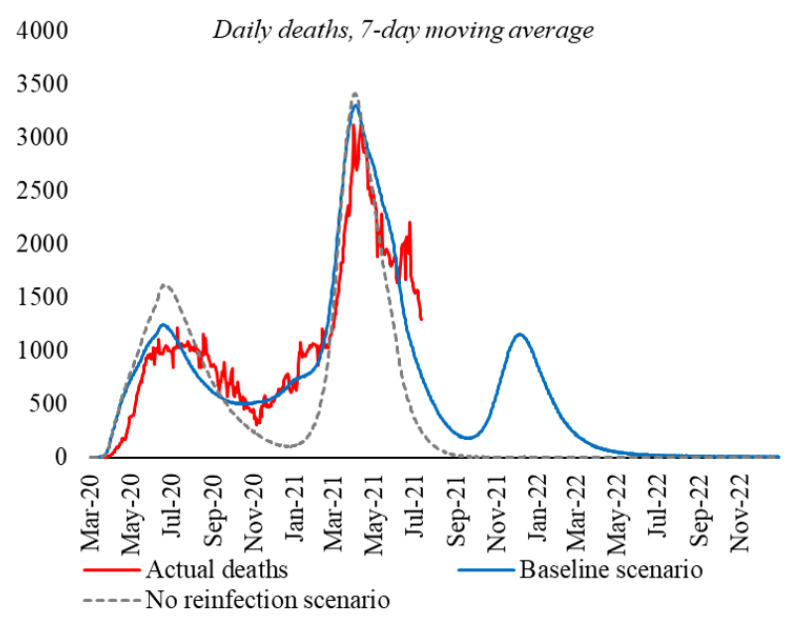

Alternatively, we can allow re-infections in the same calibrated model. In particular, we assume that people can get reinfected after 200 days from their original infection date. When reinfections are introduced this way, the $\mathrm{P} 1$ variant only needs to be 33 percent more infectious than the original COVID-19 strain to match the data. If an equally infectious third variant hits the country also in June 2021, we project a third wave, similar in size to the first wave in mid2020, but smaller than the second wave in March-April 2021. The projected vaccination path in this case is not enough to completely offset the reduced immunity in the population stemming from the possibility of reinfection. This third variant would result in 43.3 percent more deaths by the end of 2022 when re-infections happen as modelled in this section. Given the uncertainty about how re-infections happen in the real world, this scenario highlights the importance of accelerating the vaccination campaign to increase the level of immunity in the population to prevent the spread of new variants and deaths.

${ }^{13}$ There is large uncertainty about the exact number of how much more infectious the p1 is, see https://science. sciencemag.org/content/371/6526/288 for a discussion. 


\section{Super spreading variant}

Although most advanced and some emerging economies are making considerable progress in vaccinating their populations, the emergence of a new more infectious variant posses a significant risk. This risk is not just to those countries with low vaccination rates, it is a material risk to many advanced economies were vaccine hesitancy has already, or could moving forward, slow the pace of vaccinations. In this section, we use the model described in section III and the IMF's G-20 Model, Hunt and others (2015) to explore the implications of the emergence of a super infectious virus variant and its potential impact on G-20 countries epidemiological and economic outcomes.

In this scenario, we assume that new variant emerged globally all at the same time in the first days of June 2021. We also assume that this new variant is 50 percent more infectious than the UK variant (alpha), but that the mortality rate of this new variant is the same as previous virus strains. Moreover, we assume that vaccine efficacy remains the same against this new virus strain.

With respect to mobility, our model does not predict how agents would react to an increase in infection rates. Thus, in this section we just assume that people would react by lowering mobility to a point where deaths are around 20 percent lower in 2022 than they would have been if mobility remained unchanged.

In our model, we also do not model directly the relationship between mobility and domestic demand. So, for this section we assume that this relationship would be the same as the one observed during the last quarter of 2020 and the first quarter of 2021 once government spending is removed. This assumption reflects the fact that the elasticity of output to mobility declined between the first and second halves of 2020, as agents learn how to better conduct economic activity with the virus (Fig 12).

When calculating this elasticity, we also try to correct for seasonal variation. Seasonal patterns in mobility are critical, especially for the holiday season in Q4 (Fig 13). The strategy used here was to remove holiday effects from the underlying mobility series. While this does not completely correct for seasonality, it diminishes its effects. Finally, we measure the impact of mobility on domestic demand excluding government purchases and governments indirect transfers to households. The reason is that modelling fiscal policy is beyond the scope of this paper. 
Figure 12. Elasticity of Domestic Demand to Google Mobility

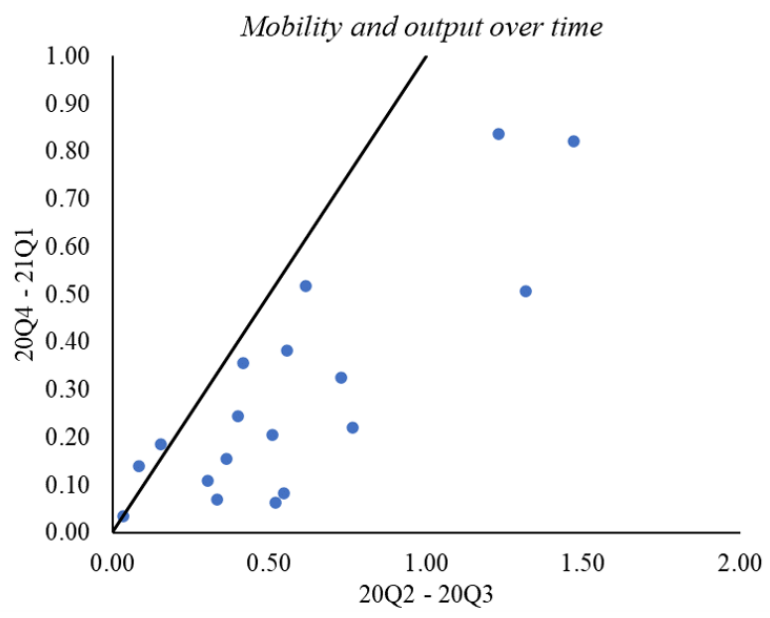

Figure 13. The Seasonality in Mobility Could Bias the Relationship With Output

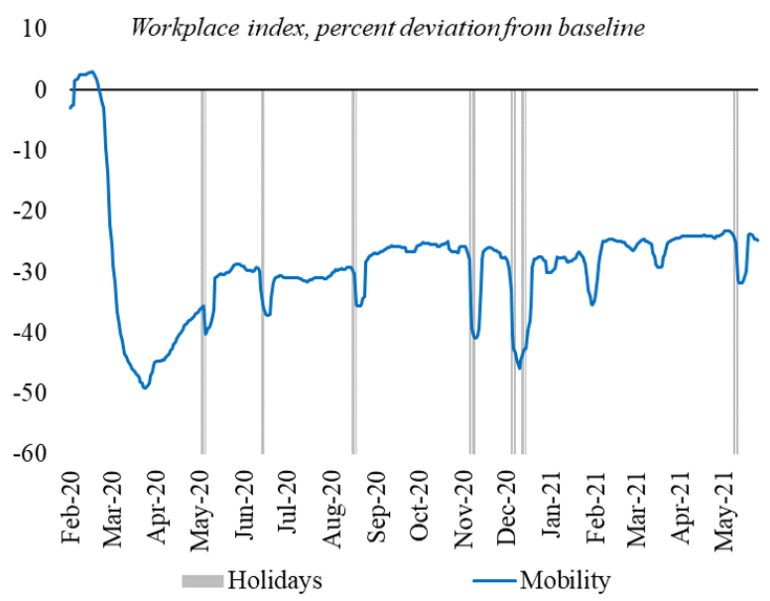

The most important factor for the output effects in this section have to do with the vaccination campaign across countries. On the supply side, even though vaccine availability has increased in the last month, vaccine supply remains a major issue in most of the developing world. While Canada has already given a first shot to close to 65 percent of its population, South Africa has vaccinated less than 10 percent of its population. The good news is that some emerging market economies have been catching up recently, with Turkey, Argentina, and Brazil already inoculating more than 30 percent of their populations (Fig 14). However, in the context of a more rapidly spreading new variant, these levels of vaccination are not enough, and most countries remain vulnerable in the near term. 
Figure 14. The Unequal Vaccine Rollout Remains a Major Issue in Most of the Developing World

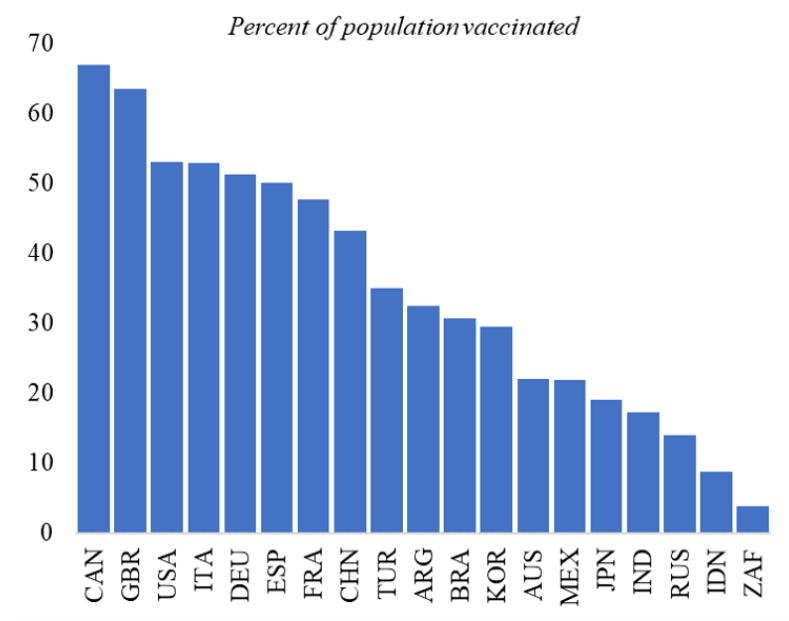

On the demand side, vaccine hesitancy might be a problem for developed countries going forward. To highlight that, we show the survey below conducted by "global data intelligence company Morning Consult" 14 in 15 countries between June 8-14, 2021 (Fig 15). Each survey respondent is asked several questions on COVID-19 vaccines. These include whether respondents would take a COVID-19 vaccine if available (or if they have already been vaccinated). Russia, Australia, South Korea, and the USA have the highest rates of people unwilling or uncertain about getting the vaccine. Meanwhile, nearly everyone in India and China is planning to get inoculated. In the context of a highly infectious COVID-19 variant, this level of hesitancy will play a central role, especially in 2022 when vaccine supply will be less of a constraint.

Among G-20 countries, India would be one of the most vulnerable countries to the emergence of a super infectious variant. In the figure below, it is assumed that India would only fully vaccinate 70 percent of its population by June 2022. The emergence of the new variant now would lead to a surge in deaths by the last quarter of 2021 and first quarter of 2022. The dotted line in fig 16 shows a path in which mobility is lowered by enough to reduce deaths by around 35 percent from the third quarter of 2021 to the end of 2022. Such a reduction in mobility would have large economic effects. If the relationship of mobility to output is similar to what it was in the last quarter of 2020 and the first quarter of 2021, Indias GDP growth could

${ }^{14} \mathrm{https}: / /$ morningconsult.com/global-vaccine-tracking/ 
Figure 15. Vaccine Hesitancy Will Play Critical Role in the Next Few Months

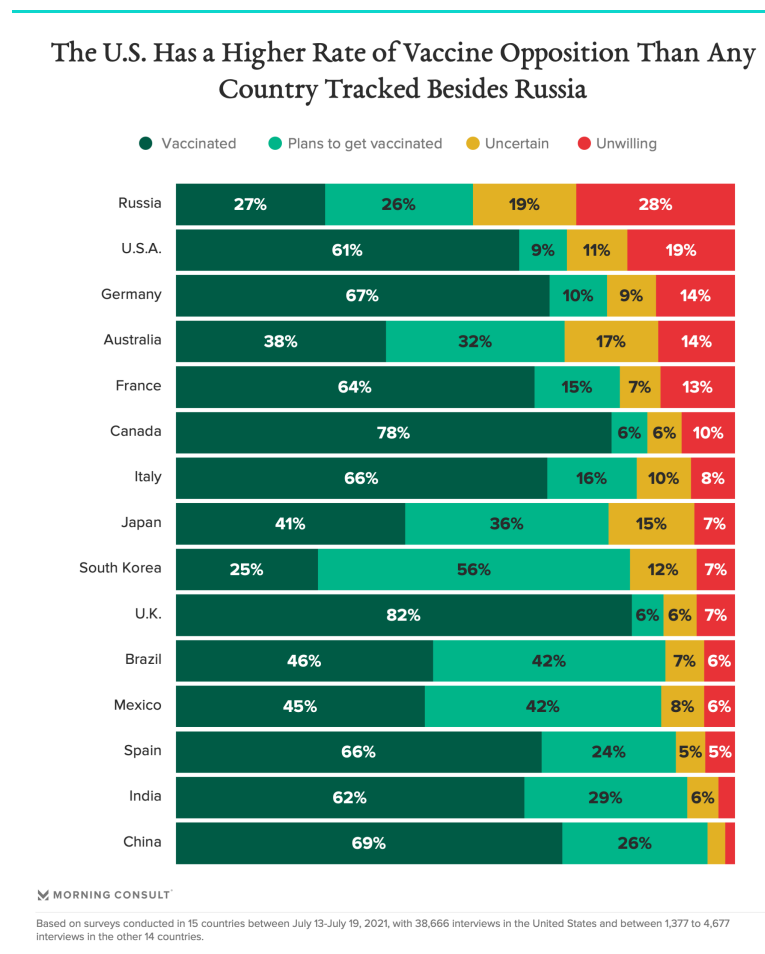

fall by around 2.4 percent percentage points in 2022 compared to the situation in which this new variant did not emerge (Fig 16).

Figure 16. India Could be Severely Affected by a More Infectious Virus Strain

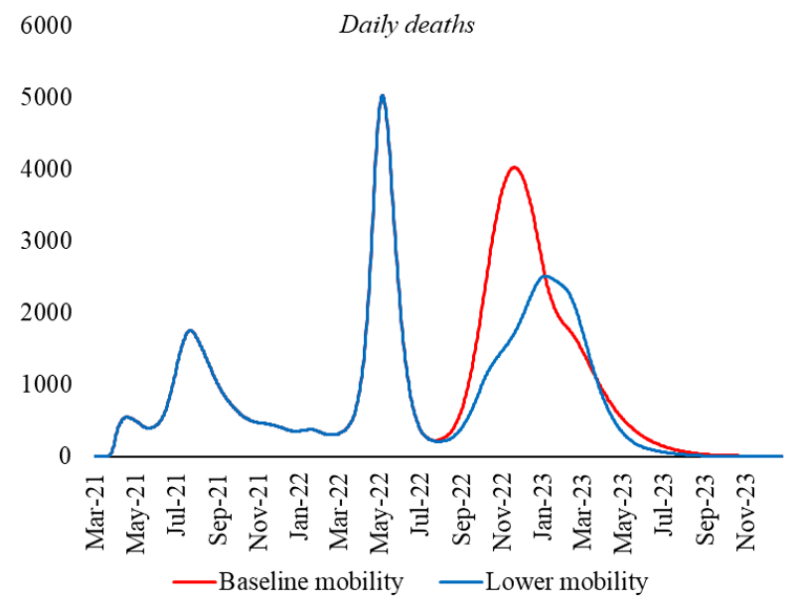


Vaccination in the USA has slowed down significantly, and vaccine hesitancy remains high. With the current vaccination levels, the US would not be immune to the fast spreading virus variant considered in this scenario. If only 60 percent of the population is vaccinated, the number of deaths would increase again late-2021 and early-2022. Even though the number of deaths would not reach the levels observed in late-2020 and early-2021, they would remain elevated for a long period (Fig 8).

If mobility were to respond so that the number of deaths were reduced by around 20 percent, the direct negative impact on the level of GDP could be as large as 0.7 percent in 2022. If global spillovers are also accounted, the negative effect could reach 0.9 percent in $2022 .{ }^{15}$ Even with this lower mobility, around 100,000 deaths would still be recorded in 2022. This dire scenario highlights the importance of addressing vaccine hesitancy and expanding vaccination to at least 70 percent of the population.

The current and projected levels of vaccination will not shield the global economy from a fast spreading virus. The rising in infections in the second part of 2021 and beginning of 2022 leads to a deterioration in activity in contact-intensive sectors, with the associated income effects spilling over to other sectors. Since emerging market economies are lagging on vaccination rollouts, the impacts of the virus variant are already felt in 2021 and remains in 2022. As for advanced economies, it would take a little longer for the increase in cases to lead to a higher impact on deaths and those would be felt in output more pronounced in 2022.

In countries with high vaccination, such as the UK and Canada, the impact would be mild, meanwhile countries lagging in vaccination, such as India and Indonesia would suffer the most among the G20 economies (Figure 17). Given the global nature of the shock, these domestic demand effects are then amplified via trade. The more protracted weakness in activity is assumed to create additional, persistent damage to economies supply capacity, with a loss in productive capital, a persistent rise in the natural rate of unemployment, and temporarily weaker productivity growth. These scarring effects are assumed to be largely felt in 2022 and beyond.

Fig 18 contains a decomposition of the impact on global GDP of the key layers of this scenario. Relative to a baseline without this virus variant, global growth in 2021 and 2022 is around 0.8 percentage points weaker. Emerging market economies excluding China are more

${ }^{15} \mathrm{Of}$ course, in this scenario the US government would most likely enact additional fiscal policy measures that would dampen the impact on the economy. 
Figure 17. Vaccination and Output Losses

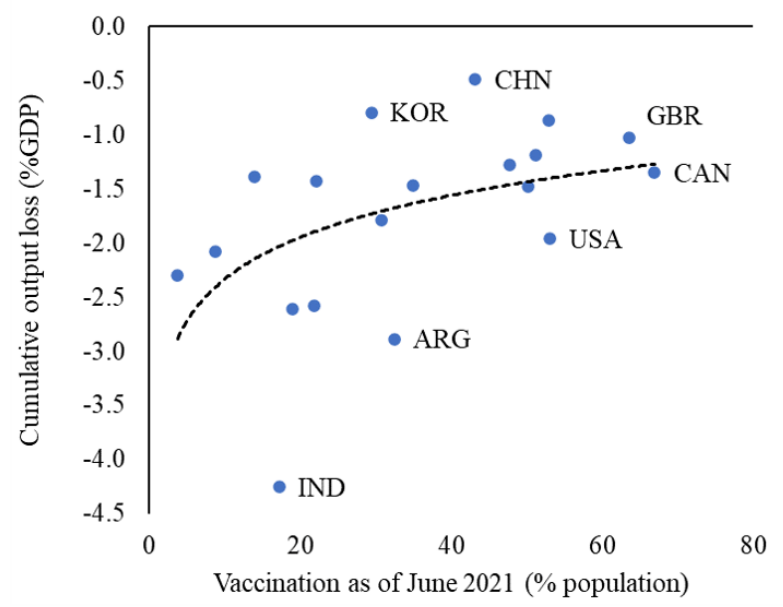

negatively affected with GDP growth below baseline by around 1.3 percentage points in 2021 and 2022. Meanwhile, vaccine hesitancy and global spillovers cost advanced economies around 0.8 percentage points in GDP growth.

In the scenario considered, the emergence of a more infectious variant results in a cumulative loss in global real GDP of 4.5 trillion US dollars over the 2021 to 2025 period. Although emerging economies excluding China see the largest percent decline in output owing to much lower vaccination rates, their real output losses are just under 2 trillion US dollars. Advanced economy losses are larger, at roughly 2.5 trillion US dollars.

\section{Conclusions}

With the emergence of new variants, the horizon to completely control the spread of COVID19 seems to have been delayed. Moreover, the vaccination campaign in several countries, especially developing countries, have yet to reach a critical mass of people to effectively end the pandemic. In this context, we presented a tool that can be used to make sense of all these new developments.

This paper introduced a simple, easily updated and close to the data model that has been used for near-term forecast and policy analysis. Even though the literature on COVID-19 has quickly grown and covers several aspects of the pandemic, with several behavioral and struc- 
Figure 18. Emerging Market Economies Could Suffer Large Output Losses
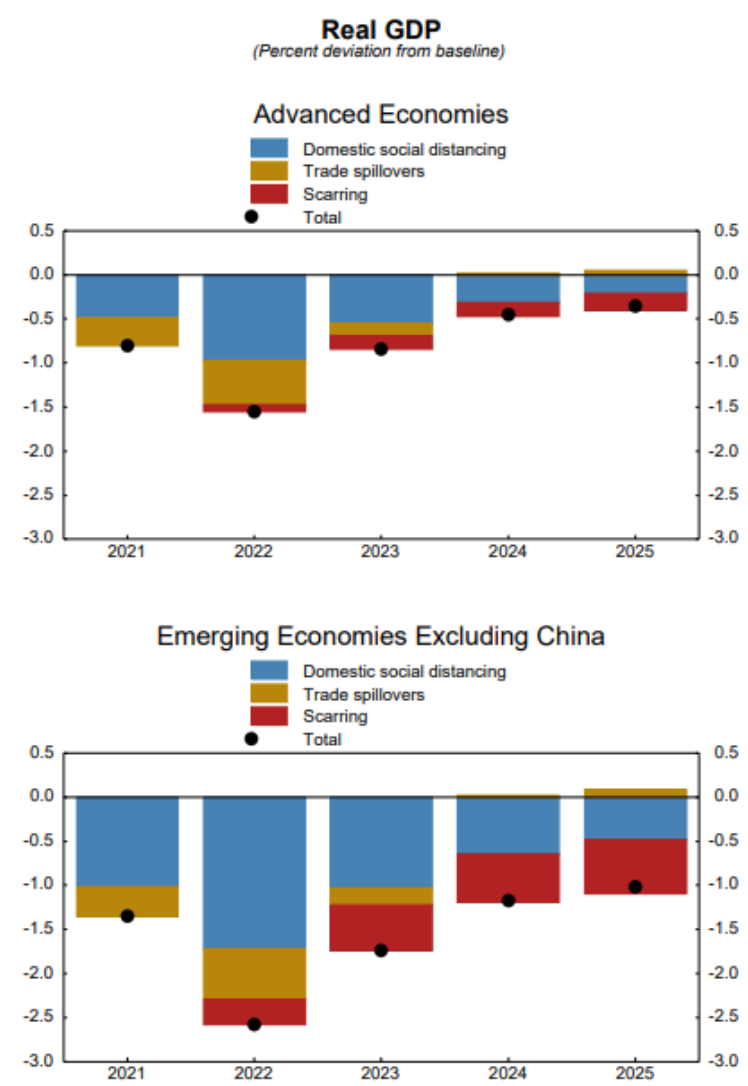

Source: IMF G20MOD simulations

tural models, it lacked a model that could be used daily to assess the state of the pandemic in simple terms and related to an important aspect of how the pandemic affects the economy, which is people's mobility.

We have shown how the model has been used to produce scenarios and form near-term virus developments forecasts. In particular, we have shown that the vaccination campaign in the UK has muted dramatically the possible effects that the highly transmissible variant alpha could have had. We have also shown how we expect a highly transmissible variant to spread in the UK depending on how transmissible it is. We showed that a high proportion of the vaccinated can be infected by the virus but deaths would not be nearly as high as previous waves because of the immunity gained from vaccines.

We have used the model calibrated to Brazil to show the importance of re-infections and discuss how it has been modeled in the literature. We still do not have enough information at a 
large scale to properly model re-infections, and that will be critical going forward in forming our forecast on how the most transmissible variants will spread in the near-term.

In our model projections, the current vaccination levels in the USA would not be enough to avert another wave of deaths later in the year. However, we showed that a minor vaccination expansion in the next few days could nearly abort the threat brought by the more infectious delta variant.

Finally, we showed how our model can be linked to other simple models to produce economic forecast and to measure the benefits and returns of vaccination campaigns. We showed that the delta variant could cost the world economy some trillions of dollars because of insufficient immunity across countries. The longer the virus is left to replicate, the more likely it is that more variants could emerge and threaten the world's economic recovery. The potential returns to further expanding vaccination production and distribution are enormous. 


\section{REFERENCES}

Atkeson, Andrew, 2020, "What Will Be the Economic Impact of COVID-19 in the US? Rough Estimates of Disease Scenarios," Working Paper 26867, National Bureau of Economic Research.

Bakker, Bas B, and Carlos Goncalves, 2021, "COVID-19 in Latin America; A High Toll on Lives and Livelihoods," Techn. rep., International Monetary Fund.

Berger, David, Kyle Herkenhoff, Chengdai Huang, and Simon Mongey, 2020, "Testing and reopening in an SEIR model," Review of Economic Dynamics.

Bognanni, Mark, Douglas Hanley, Daniel Kolliner, and Kurt Mitman, 2020, "Economics and Epidemics: Evidence from an Estimated Spatial Econ-SIR Model," Finance and Economics Discussion Series 2020-091, Board of Governors of the Federal Reserve System (U.S.).

Dabla-Norris, Era, Hibah Khan, Frederico Lima, and Alexandre Sollaci, 2021, "Who Doesn't Want to be Vaccinated? Determinants of Vaccine Hesitancy During COVID-19," Techn. rep., International Monetary Fund.

Dizioli, Allan, and Roberto Pinheiro, 2020, "Information and Inequality in the Time of a Pandemic,” IMF Working Papers 20/188, International Monetary Fund.

Eichenbaum, Martin S, Sergio Rebelo, and Mathias Trabandt, 2020a, “The macroeconomics of epidemics," Techn. rep., National Bureau of Economic Research.

__ 2020b, “The Macroeconomics of Testing and Quarantining," Techn. rep., National Bureau of Economic Research.

Fernández-Villaverde, Jesús, and Charles I Jones, 2020, "Estimating and Simulating a SIRD Model of COVID-19 for Many Countries, States, and Cities," Techn. rep., National Bureau of Economic Research.

Fernández-Villaverde, Jesús, and Charles I Jones, 2020, "Estimating and Simulating a SIRD Model of COVID-19 for Many Countries, States, and Cities," Working Paper 27128, National Bureau of Economic Research.

Ganslmeier, Michael, Pragyan Deb, Davide Furceri, Jonathan Ostry, and Nour Tawk, 2021, "Vaccinate Early and Vaccinate Broadly: On the health and Economic Effects of COVID19 Vaccines," .

Google LLC, 2021, “Google COVID-19 Community Mobility Reports,” Available at https: //www.google.com/covid19/mobility/data_documentation.html (2021/07/07).

Hannah Ritchie, Diana Beltekian Edouard Mathieu Joe Hasell Bobbie Macdonald Charlie Giattino Cameron Appel Lucas Rodés-Guirao, Esteban Ortiz-Ospina, and Max Roser, 2020, "Coronavirus Pandemic (COVID-19)," Our World in Data, https://ourworldindata.org/coronavirus. 
Hunt, Mr. Benjamin L, Mr. Douglas Laxton, Mr. Dirk V Muir, Ms. Keiko Honjo, Michal Andrle, Susanna Mursula, René Lalonde, Stephen Snudden, Mika Kortelainen, Patrick Blagrave, and Pedro Espaillat, 2015, "The Flexible System of Global Models FSGM," IMF Working Papers 2015/064, International Monetary Fund.

Kermack, W. O., and A. G. McKendrick, 1927, "Contributions to the mathematical theory of epidemics, part i," Proceedings of the Royal Society of Edinburgh. Section A. Mathematics, Vol. 39, No. 3, p. 700721.

MacIntyre, C Raina, Valentina Costantino, and Mallory Trent, 2020, "Modelling of COVID19 vaccination strategies and herd immunity, in scenarios of limited and full vaccine supply in NSW, Australia," medRxiv.

McCarthy, Niall, 2020, “The Countries with the Most Critical Care Beds per Capita," Techn. rep., Statista Inc.

Moore, Sam, Edward M. Hill, Louise Dyson, Michael J. Tildesley, and Matt J. Keeling, 2021, "Modelling optimal vaccination strategy for SARS-CoV-2 in the UK," PLOS Computational Biology, Vol. 17, No. 5, pp. 1-20.

National Health Service, 2021, "COVID-19 Vaccinations," Available at https://www.england. nhs.uk/statistics/statistical-work-areas/covid-19-vaccinations/ (2021/07/15).

Odone, Anna, Davide Delmonte, Thea Scognamiglio, and Carlo Signorelli, 2020, "COVID19 deaths in Lombardy, Italy: Data in context," The Lancet Public Health.

Ward, Helen, Christina Atchison, Matthew Whitaker, Kylie EC Ainslie, Joshua Elliott, Lucy Okell, Rozlyn Redd, Deborah Ashby, Christl A Donnelly, Wendy Barclay, Ara Darzi, Graham Cooke, Steven Riley, and Paul Elliott, 2020, "Antibody prevalence for SARSCoV-2 following the peak of the pandemic in England: REACT2 study in 100,000 adults," medRxiv. 